\title{
Il packaging come icona culturale? Processi di significazione e meccanismi intertestuali
}

\author{
Ilaria Ventura Bordenca \\ Università degli Studi di Palermo, Dipartimento Culture e Società, Palermo \\ ilaria.venturabordenca@gmail.com
}

\begin{abstract}
This paper analyses product packaging as cultural icons that condense and spread socio-cultural values. Some global brand's packs became unmistakable signs of those brands (take the contour bottle of Coca-Cola or the Nutella jar) but also objects able to communicate consumption values, cultural habits and collective usages. Through the analysis of some case studies from the fields of branding and marketing, this paper aims at investigating the semiotic processes that transform packaging from commercial and common objects to cultural icons. The main idea is that intermedial translations and interdiscoursive migrations are the fundamental mechanisms involved in this processes of iconization (with specific packaging items moving from the field of branding to those of arts, music, movies, design, fashion).
\end{abstract}

\section{Keywords}

Packaging; Intertestuality; Translation; Brand; Visuality

\section{Sommario/Content}

1. Oggetti innocenti

2. Interdiscorsività, andata e ritorno

3. Icone culturali e usi collettivi

4. Apparenti contrasti: astrazione visiva ed effetto di iconicità

5. Conclusioni

Bibliografia 
Vol 21, No 22 (April 2020) • DOI: 10.12977/ocula2020-20

Ilaria Ventura Bordenca | Il packaging come icona culturale? Processi di significazione e meccanismi intertestuali

\section{Oggetti innocenti}

Barthes (1957) scriveva che il mito è "parola innocente", ovvio, naturale. Ecco perché si potrebbe difficilmente obiettare che la bottiglietta della Coca-Cola o il barattolo di Nutella non siano, in qualche modo, miti della contemporaneità. Oggetti specificatamente pensati dal marketing, alcuni packaging, infatti, ad un certo punto della loro storia diventano altro, iniziano a significare molte più cose di quelle che ci si aspetterebbe da un barattolo o da una bottiglia, marchiati e progettati, in linea di principio, solo per promuovere un prodotto e il suo brand (Ferraresi 1999; Bucchetti 1999, 2005; Ventura 2014). Ce lo dice la società, attraverso vari e diversi generi discorsivi: riviste, giornali, libri, siti web, mostre, albi illustrati, raccontano la storia di certe confezioni, ne narrano le origini - quasi sempre avvolte in un'aura di mistero - e i vari restyling, il legame con la società di massa, con la cultura popolare, con attori, sportivi, cantanti, e vari personaggi che, più o meno consapevolmente, vi hanno fatto da testimonial. Esitono libri, ${ }^{1}$ pubblicati da storici, giornalisti, studiosi, su Nutella e il suo barattolo Pelikan, ${ }^{2}$ su Coca-Cola e le fortune della bottiglia contour, ${ }^{3}$ sul mondo Pepsi ${ }^{4}$ e su altri brand famosi, tra le varie cose, anche per un packaging memorabile. Ci sono albi illustrati che celebrano i legami tra marche e cultura pop: tra le più recenti, del 2013, la raccolta fotografica di Assouline, Coca-Cola: Film, Music and Sports, in cui compaiono, tra gli altri, Elvis Presley, Marilyn Monroe, Chewbecca, Whitney Houston, Jesse Owens. Sui marchi, i loro oggetti e la loro storia, si organizzano anche mostre (come "The Coca-Cola bottle. An american bottle at 100", organizzata ad Atlanta nel 2015, per il centenario della bottiglia) e raccolte complete delle apparizioni cinematografiche dei brand commerciali. Wikipedia, interrogato sulla storia di un certo marchio, ne elenca anche le citazioni in film, musica, cinema, arte, design, moda ecc. In tutti questi tipi di testi, le immagini di

1 Tra cui, in Italia, quelli di Gigi e Clara Padovani, come ad esempio Mondo Nutella (2014, Rizzoli), Pane e Nutella (2012, Giunti), Passione Nutella (2010, Giunti), 40 ans de plaisir (2005, Michel Lafon), Gnam storia sociale della Nutella (1999, Castelvecchi), Nutella un mito italiano (1998, Rizzoli); i libri comici di Riccardo Cassini Nutella Nutellae (2001, Panini), Nutella 2 La Vendetta (1993, Panini) e Il piccolo libro della Nutella (2000, Mondadori).

2 L'attuale confezione è stata introdotta negli anni Sessanta e pare che sia stata chiamata così perché ricorda il calamaio d’inchiostro Pelikan.

3 Termine francese per "sagoma". Quando venne progettata, nel 1915, questa bottiglia era stata pensata proprio per essere inconfondibile e immediatamente risconoscibile alla vista e al tatto. Come per ogni oggetto mitico, di cui si narrano origini leggendarie, non si sa a cosa sia stata ispirata: se alle hobble skirts delle dame di fine Ottocento, se a un baccello di cacao, o se alla bocca di Mae West. Non importa stabilire una vera storicità di queste ipotesi, importa che esistano. Il numero di libri su Coca-Cola non si contano. Qui citiamo soltanto Kiss the Past Hello. 100 Years of the Coca-Cola Bottle, una raccolta fotografica di citazioni e riusi artistici della bottiglia, edita da Assouline, e The Coca-Cola Bottle, sorta di manuale per collezionisti.

4 Molto meno celebrata e studiata della concorrente, ma presente sul mercato editoriale soprattutto americano sia con volumi per collezionisti sia con volumi celebrativi come Pepsi: 100 years. 
Vol 21, No 22 (April 2020) • DOI: 10.12977/ocula2020-20

Ilaria Ventura Bordenca | Il packaging come icona culturale? Processi di significazione e meccanismi intertestuali

accompagnamento che ritornano, accanto al logo, sono proprio quelle delle confezioni: bottiglie, barattoli, scatole e quant'altro. Certamente un tal tipo di produzione editoriale e culturale è in parte frutto di un lavoro di marketing (spesso le mostre sono eventi celebrativi organizzati dal brand stesso), ma può anche essere intrepretata come il riconoscimento esplicito che quel brand, con i suoi prodotti e i suoi oggetti identificativi, ha assunto nel tempo significati ulteriori a quelli strettamente commerciali.

Una icona culturale si definisce in base alla sua "portabilità" in vari campi e in numero alle sua trasformazioni in vari contesti comunicativi e culturali, con una sovrapposizione di livelli "alti" e "bassi" della cultura (Fiorentino 2009). Alcuni packaging, che sono oggetti progettati con un fine pratico (conservare e trasportare un prodotto) e un fine comunicativo (raccontare il prodotto e promuovere il marchio di appartenenza), rispondono a questa definizione in quanto assurgono a un ruolo culturale più ampio. Essi, infatti: (i) migrano, dal marketing e dal branding, nei campi della moda, dell'arte, del design, del cinema, dello sport, producendo significazioni nuove, senza perdere però riconoscibilità; (ii) parallelamente a questo processo di migrazione, vengono indicati dal discorso sociale stesso come icone, ovvero come qualcosa che è in grado di sintetizzare brevemente e immediatamente una serie di significati culturali di più ampio respiro, una intera comunità, lo spirito di un'epoca, un periodo storico.

Panofsky mettendo a punto la differenza tra iconologia e iconografia chiariva proprio che, a differenza della seconda, che è analitica e descrittiva, la prima si occupa di dare una visione di sintesi dei significati delle immagini, esplicitandone i contenuti ideologici, filosofici, storici, che rappresentano «le tendenze essenziali dello spirito umano» e che si possono individuare tramite un' «intuizione sintetica» (1995: 44, tr. it.). Eco, apprestandosi all'analisi delle strisce a fumetti di Superman, sosteneva che il processo di mitizzazione è «simbolizzazione inconscia, identificazione dell'oggetto con una somma di finalità non sempre razionalizzabili, proiezione nell'immagine di tendenze, aspirazioni, timori particolarmente emergenti in un individuo, in una comunità, in una intera epoca storica» (1964: 221).

Ecco che, tornando ai packaging, essi sarebbero in grado, attraverso una serie di migrazioni, usi e trasformazioni tra vari campi culturali e tra vari media, di funzionare come icone, sintesi visive di una batteria ampia di significati socio-culturali.

Se la produzione mediatica e culturale, in senso ampio, celebra - e con ciò esplicita - tale ruolo, sono anche le marche stesse, che dei packaging sono il principale Enunciatore, a dire, nella propria comunicazione promozionale, quanto essi non siano semplicemente oggetti di mero scambio economico o consumo commerciale, ma segni di una cultura. Si sa, tra l'altro, che vi sono marchi che fanno della rottura dei confini commerciali e della migrazione in altri campi uno dei propri tratti distintivi, sfruttato e ampiamente riutilizzato sia dal marchio stesso sia da altre sfere della cultura, in un scambio fluido, continuo e reciproco (Marrone 2007).

Sul sito web italiano dell'acqua di lusso Perrier, ad esempio, la sezione dedicata alla storia dell'azienda si intitola "Un marchio francese divenuto icona", 


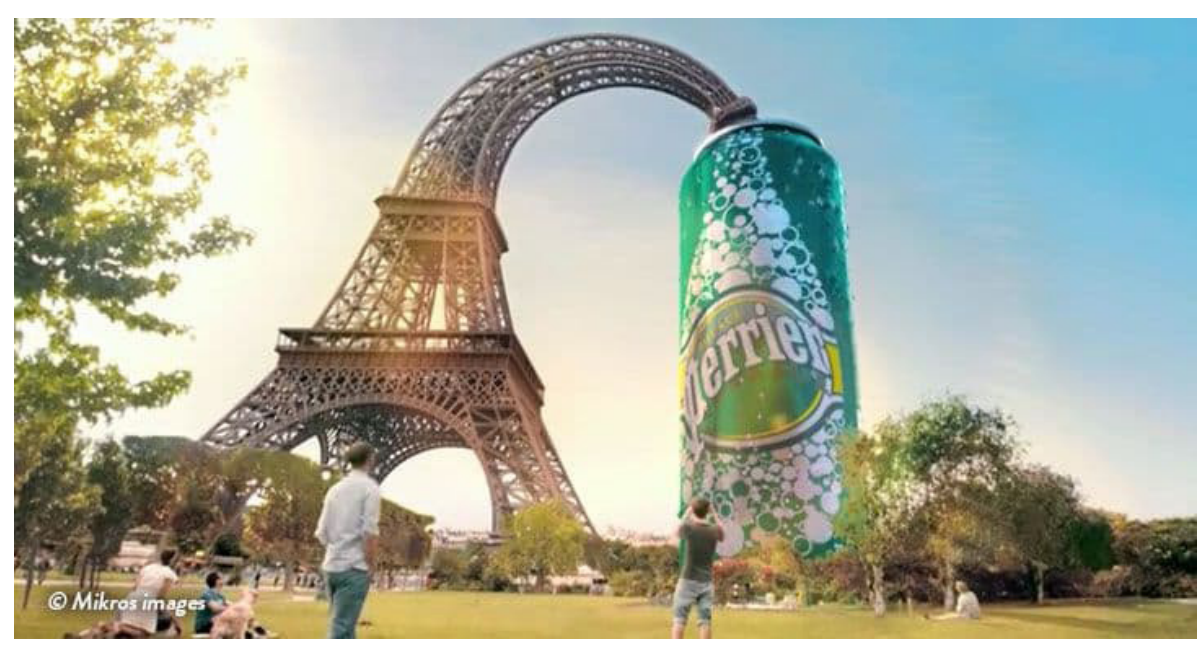

Figura 1. Francesità <www.perrier.com/it>.

con lo sfondo di una Tour Eiffel che "beve" - francesità, evidentemente - da una lattina (fig. 1). L'account Instagram di Perrier, inoltre, è incentrato sull'estetizzazione della bottiglia e gioca continuamente con la parola "icona" ("Mosaiconic", "Iconic Bubble", "Iconic Bottle").

Un tal processo di riappropriazione e rilancio, da parte delle marche, delle confezioni dei prodotti come icone si basa su un rapporto dialogico, fitto e inevitabile, che intercorre tra discorso proprio della marca e altri discorsi sociali (Marrone 2007): i marchi si nutrono e si alimentano continuamente degli accostamenti e delle relazioni con personaggi, situazioni, contesti, ciascuno con i suoi propri significati ed effetti. Un conto sarà la foto della Coca-Cola con Elvis, un conto la foto con Jesse Owens. Un altro ancora l'effetto di senso globale dell'albo fotografico sulle relazioni tra Coca-Cola e l'arte, la musica e lo sport, nel suo complesso, per il solo fatto che esiste.

Un simile intreccio di testi, di marca e non di marca, conduce alla produzione di una macrosfera composta da una pluralità di linguaggi espressivi (visivo, verbale, spaziale ecc.) e di generi (giornalistico, pubblicitario, televisivo, artistico, ecc.) all'interno del quale il packaging trova posto e si muove, migrando da un discorso all'altro.

Tale macrosfera di testi funziona per noi come un metalinguaggio: testi che parlano di altri testi - i packaging e le loro marche, in questo caso - e ci illustrano così le regole per la loro interpretazione e, al tempo stesso, per l'interpretazione della nostra cultura. Barthes (1957) definisce il funzionamento del mito proprio come un metalinguaggio.

Da questo punto di vista, concepiamo l'icona culturale come un concetto assumibile nella semiotica della cultura così come elaborata da Lotman (1973, 1980, 1983, 1985), nello specifico con riferimento all'idea che ogni testo può essere inteso come la condensazione di una intera cultura - tant'è che Lotman lavorava anche su manifestazioni piccole, su microconfigurazioni (singole poesie, balletti, stili di comportamento ecc.) da cui si poteva ricostruire lo spirito di una intera comunità, ovvero un intero sistema di valori. 
Vol 21, No 22 (April 2020) • DOI: 10.12977/ocula2020-20

Ilaria Ventura Bordenca | Il packaging come icona culturale? Processi di significazione e meccanismi intertestuali

È quello che accade effettivamente con il fenomeno delle icone culturali: singoli oggetti, frammenti di storie, saghe mediatiche, personaggi, merci, logo, singole parole, che rappresentano molto altro, e acquisiscono significati diversi e più ricchi di quelli che avrebbero in teoria nel loro contesto di partenza (si pensi alle mitologie di Superman, del Titanic, e perfino a quella di Ikea $^{5}$ ). La caratteristica propria dell'icona culturale, quella di rappresentare brevemente un qualche tipo di cultura e quindi di funzionare come una parte per il tutto, si collega a ciò che scrive sempre Barthes: nel mito

il concetto può espandersi attraverso un'estensione molto grande di significante: in tal caso, ad esempio, un libro intero potrà essere significante di un solo concetto; e, inversamente, una forma minuscola (una parola, un gesto, anche marginale purché sia notato) potrà servire come significante di un concetto gonfio di una ricchissima storia. (Barthes 1957: 202)

Nello specifico, chiedendoci in che modo le confezioni dei prodotti di alcuni brand mondiali possano avere assunto un tale ruolo nella società, assumiamo che mito possa essere qualsiasi cosa per il fatto che esso non è una cosa, bensì un messaggio, un sistema di comunicazione, una forma: «Tutto dunque può essere mito? [...] Ogni oggetto può passare da un'esistenza chiusa, muta, a uno stato orale, aperto all'approvazione della società» (Barthes 1957: 191, tr. it.).

È l'uso sociale e storico che si fa di certi oggetti del mondo ad assumerli come miti. L'effetto finale, a livello di senso comune, è l'idea barthesiana del mito come natura, fatto ovvio, indiscutibile tale per cui tutti possano dire di un certo oggetto che esso è simbolo di qualcosa. Il processo sotteso a tale naturalizzazionse, che trasforma ciò che è Storia in fatto di Natura, è dato per scontato, obliato.

Obiettivo di questo lavoro è capire qual è il tipo di uso sociale e culturale che mitizza certi oggetti del mondo di marca, nello specifico quali sono i processi di trasformazione di alcuni packaging, banali contenitori di qualcosa, in icone culturali.

L'ipotesi è che siano i movimenti intertestuali e interdiscorsivi a creare l'icona culturale come effetto, e che nel corso di questo processo il packaging subisca anche una semplificazione visiva, la quale, piuttosto che affievolirne il potere semiotico, ne rafforza l'identità. Un'altra caratteristica dell'icona culturale è infatti proprio il mantenimento di alcuni tratti invarianti di base che ne garantiscono la riconoscibilità (Fiorentino 2009).

Prenderemo in considerazione alcuni brand internazionali (Coca-Cola, Pepsi, Perrier, Nutella), selezionati, tra tutti quelli possibili, per tre ragioni. Primo, perché si tratta di brand i cui packaging sono noti, nel senso comune, per essere vere e proprie icone, riconosciute come tali dalla società, con una serie di testi che parlano di esse come esplicitamente capaci di significare molto altro. Tale riconoscimento sociale vale per noi, però, come punto di parten-

5 Su Superman cfr. in primis Eco (op. cit.), sulla generazione del mito mediatico di Titanic cfr. Heyer (2012), sul mito Ikea cfr. Mangano (2014, 2019). 
Vol 21, No 22 (April 2020) • DOI: 10.12977/ocula2020-20

Ilaria Ventura Bordenca | Il packaging come icona culturale? Processi di significazione e meccanismi intertestuali

za per l'analisi, come la punta di un iceberg. In secondo luogo, per una ragione per così dire quantitativa, e cioè perché effettivamente, compiendo una prima esplorazione di ampio respiro su quelli che sono i rapporti storicamente intercorsi tra branding e altri campi culturali, alcuni nomi sembrano ritornare in maniera ossessiva. E tra questi ci sono proprio i marchi a cui attingeremo come esempi per questo saggio. Terzo, perchè per l'identità, visiva e valoriale, di questi brand il packaging ha svolto un ruolo fondamentale, fin dalle origini della progettazione dei primi prototipi di confezione.

\section{Interdiscorsività, andata e ritorno}

Se fin dall'inizio non si fosse dotata di una bottiglia trasparente che mostra l'inconfondibile colore scuro della sua bevanda, l'immagine della Coca-Cola sarebbe stata la stessa? La trasparenza dell'involucro, da sola, non sarebbe sufficiente a identificare il prodotto, perché l'etichetta e la sagoma della bottiglia hanno il ruolo fondamentale di rendere riconoscibile il marchio, che a sua volta non è scindibile dalla bevanda in sé. La bevanda è il marchio, ed ha bisogno di un contenitore che manifesti concretamente i segni visivi di quel marchio. In generale, il packaging è al tempo stesso l'involucro, con proprie e specifiche caratteristiche (dimensione, sagoma, materiale, etichette, colori, nome del brand ecc.) e il prodotto che contiene. Ciò vale per qualsiasi prodotto di marca, per il semplice fatto che il contenitore non ha senso di per sé ma perchè è inserito all'interno dell'enunciazione del brand.

Il packaging è un oggetto che ha necessità di inserirsi in una rete di relazioni, per attivare i propri significati. ${ }^{6} \mathrm{E}$, come ogni testo di una data cultura, ha bisogno di dialogare con una pletora di altri testi, ovvero con la cultura stessa.

Coca-Cola è uno dei marchi che può vantare ad oggi una presenza sui media, a livello mondiale, così elevata da essere difficilmente replicabile. Le citazioni del brand, sotto forma di affissioni, logo o immagini del packaging non si contano, e sono proprio un aspetto della comunicazione di brand su cui Coca-Cola punta molto: il fatto che si tratti molto di più di un semplice marchio di bevande, ma dell'incarnazione di un certo modo di vivere, collegato all'americanità, prima di tutto, ma anche a una certa universalità dei consumi, e all'idea stessa di un consumo facile e disimpegnato (alcuni dei possibili percorsi tematici legati al nome e all'immagine di Coca-Cola).

Si pensi al cinema. Una delle prime cose che Elliot fa bere a E.T. è proprio una Coca-Cola (E.T., 1982, Steven Spielberg): perché rappresenta una cosa che lui conosce e che vuole far conoscere al suo amico alieno, e che sintetizza un pezzo di vita quotidiana americana, ma anche di abitudini umane. In $M a$

6 L'idea di oggetto su cui ci basiamo discende, tra le altre, da Fabbri (1998), secondo cui gli oggetti sono l'esito dell'incontro tra le parole e le cose che fa sì che la materia del mondo entri in contatto con una forma, una griglia culturale, trasformandosi in sostanza. Gli oggetti non sono muti, ma sono già significanti perché inseriti in una rete di relazioni e valorizzazioni sia rispetto ai soggetti sia rispetto ad altri oggetti. Sulla semiotica degli oggetti, cfr. Deni (2002), Landowski e Marrone (2002), Mattozzi (2006), Mangano (2009). 
Vol 21, No 22 (April 2020) • DOI: 10.12977/ocula2020-20

Ilaria Ventura Bordenca | Il packaging come icona culturale? Processi di significazione e meccanismi intertestuali

che siamo tutti matti? (Jamie Uys, 1980) la bottiglietta di Coca-Cola che cade dal cielo in un villaggio di boscimani, creando scompiglio e degenerazioni in una comunità che non conosceva la legge del possesso, invece, incarna i significatinegativi della civiltà occidentale consumistica e individualista. Tant'è che il protagonista deve disfarsene per far ritornare l'equilibro nel villaggio.

La scena in cui Warren Beatty e Faye Dunaway si rinfrescano in Bonnie and Clyde bevendo da una bottiglia di Coca-Cola (Arthur Penn, 1967) invece più che di valori socio-culturali ci parla di atteggiamenti, posture, effetti somatici (rinfrescarsi) e, in quella scena, delle conseguenze sensuali che ciò ha sui due protagonisti. Prima ancora, in Prima Vittoria (Otto Preminger, 1965), durante l'attacco di Pearl Harbor, il comandante della marina americana, interpretato da John Wayne, beve Coca-Cola dalla contour e nel frattempo impartisce ordini militari. E così in altre pellicole, la Coca-Cola ristora e tira su, sostiene durante le fatiche e le decisioni, esattamente come prometteva di fare nella prima metà del Novecento, presentandosi come bevanda tonificante. ${ }^{7}$ Mentre altrove, la Coca-Cola "ristora" non nel senso che tira su ma piuttosto che placa gli animi, come nel film Urla del silenzio (Roland Joffé, 1984) in cui bere la bevanda tutti insieme è segno di condivisione e riappacificazione tra giornalisti occidentali e khmer rossi.

Questi appena citati sono casi del cosiddetto product placement, una tecnica di marketing che serve a piazzare il prodotto dentro un film o un'opera editoriale, in nome di una sorta di coerenza tra storia raccontata e valori di marca. Ma il product placement fa molto di più: arricchisce di un certo senso la storia del film, o un suo frammento, grazie a un elemento che è già significante di per sé.

Nell'arte, come nel mondo cinematografico, le citazioni di Coca-Cola sono moltissime. Già Salvador Dalì inserì, nel 1943, in Poetry of America, una bottiglia di Coca-Cola in liquefazione. Fu poi con i movimenti new dada e pop art (Rauschenberg prima, poi Johns, Warhol e Wesselman) che la bottiglia e il marchio di Coca-Cola - insieme ad altri oggetti di uso quotidiano, ad altri brand e ad altri packaging (si vedano ad esempio le still life di Wesselman) - diventano figure altamente significative, usate nel discorso artistico per polemizzare sulla società di massa, sulle modalità di produzione culturale, sulla standardizzazione e il consumismo, e sull'arte stessa. La bottiglia viene modificata nei colori, moltiplicata (Green Coca-Cola Bottles 1962, Three Cokes Bottles 1962, di Warhol), apposta in nuovi contesti (come in Coca-Cola Plan di Rauschenberg, 1958) (figg. 2-5), trasformata nella sua identità in quanto, da oggetto di consumo quotidiano, diventa parte integrante di un discorso altro "alto" potremmo dire - quello artistico. ${ }^{8}$ Esce dalle case e dai supermercati per

7 Come si sa, Coca-Cola è stata inventata da un farmacista, John Pemberton, nel 1886, proprio come un liquido con azioni tonificanti, da ingerire contro malanni come il mal di testa. Era, insomma, all'origine un medicinale, secondo quelli che erano allora i canoni di definizione dei farmaci e della medicina in generale.

8 Barthes scrive a questo proposito: «Ciò che la pop art vuole è desimbolizzare l'oggetto, dargli l'opacità e l'ottusa caparbietà di un fatto [...] la pop art mette in scena una qualità filosofica delle cose chiamata fatticità: il fattico è il carattere di ciò 


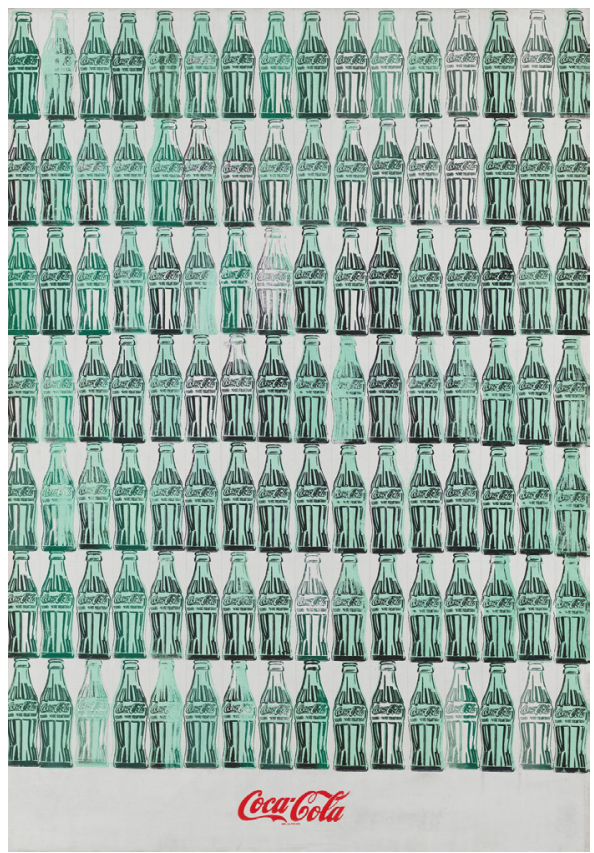

Figura 2. Andy Warhol, Green Coca-Cola Bottles, 1962.

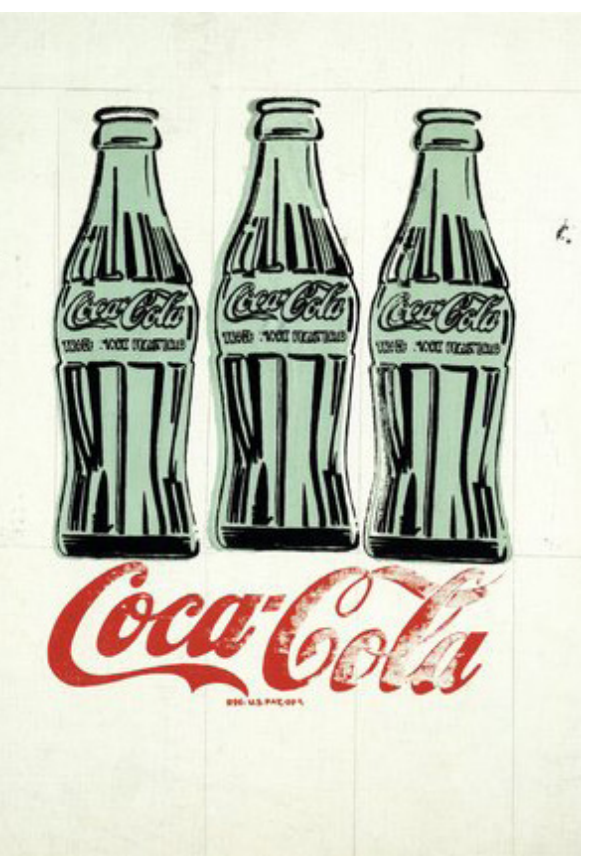

Figura 3. Andy Warhol, Three Coke Bottles, 1962.

entrare nelle gallerie e nei musei. O nelle gallerie che sembrano supermercati, come fece Warhol alla Stable Gallery di New York, nel 1964, con le Scatole di Brillo e altri prodotti, allestendo una mostra che simulava un magazzino di merci, con scatole impilate e stipate a tal punto da rendere in alcuni punti appena possibile il passaggio. La presenza della bevanda di Atlanta nell'arte è continuata nel tempo, come dimostrano le opere d'arte contemporanea in cui continua a comparire (in quelle di Mario Schifano e Mimmo Rotella, solo per citarne pochissime). Nelle opere più recenti, Coca-Cola entra non solo in quanto prodotto di consumo di massa ma anche in qualità di oggetto già citato nei movimenti artistici precedenti, e dunque carico di sensi ulteriori (fig. 5).

Se è vero che i packaging entrano nelle storie e negli altri discorsi rigenerandosi e riproponendosi sotto altre vesti, lo fanno in qualità di segni che sono già potenti a livello culturale. Come abbiamo avuto modo di dire altrove (Ventura 2014), le riproposizioni artistiche o mediatiche di segni commerciali come logo o packaging non vanno intese come attività che rendono icona qualcosa che ancora non lo è, ma utilizzano e rimaneggiano simboli già carichi a livello semantico, anzi sono proprio questi movimenti interdiscorsivi che ne svelano

che esiste in quanto fatto e appare sprovvisto di qualsiasi giustificazione: gli oggetti raffigurati dalla pop art non sono solamente fattici, essi incarnano anche il concetto stesso della fatticità - e qui, loro malgrado, ricominciano a significare: essi significano che non significano niente» (Barthes 1982: 198-199, tr. it.). Barthes intende dire che le confezioni ritratte da Warhol o da Rauschenberg sono messe in grado di significare un'apparente loro ottusità proprio grazie al processo enunciativo artistico specifico della pop art. 


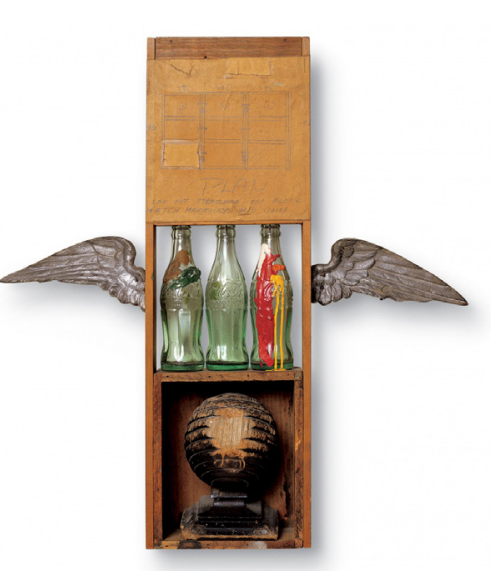

Figura 4. Coca-Cola Plan, Robert Rauschenerg, 1958.

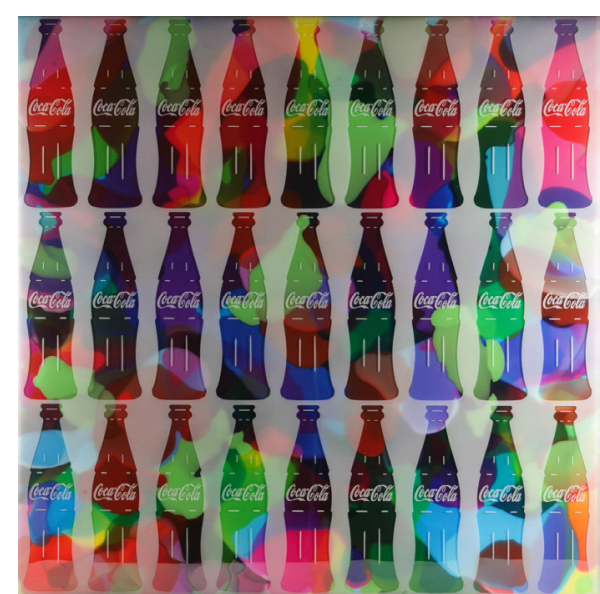

Figura 5. Always Coca-Cola, Alberto Murilio, 2014.

l'iconicità. Se da una parte dunque l'icona culturale si definisce per il fatto di migrare da una sfera a un'altra, circolando e diffondendosi anche, e soprattutto, a livello del senso comune, bisogna considerare il fatto che ciò che assurge a icona è già socialmente determinato, non è affatto neutro. Cioè, se Coca-Cola viene usata dall'arte o dal cinema per sintetizzare una certa cultura di massa, per significare un'epoca o un modo di vivere, e attraverso, una vera e propria traduzione interdiscorsiva, si trasforma al punto da essere in grado, all'interno di una catena intertestuale e intermediale, ${ }^{9}$ di farsi portatrice di altri significati, a volte critici e di contestazione, è perché è già essa stessa carica di significati. I quali derivano dalla pubblicità, in primis, ma anche dall'uso quotidiano, dal passaparola, dall'humus culturale, dai passaggi negli altri discorsi sociali.

Prendiamo adesso il caso di Nutella, ormai da tanti anni brand internazionale. Nutella e il campo del design si sono sempre stretti la mano, lo testimoniano, nel 2018, il German Design Award per la riprogettazione del suo celebre barattolo in vetro, e, nel 2014, il cobranding con Alessi per il Nutella Clock (fig. 6), oltre a varie collaborazioni con designer di tutto il mondo per ripensare non solo il barattolo, ma anche tutto l'insieme di oggetti che vi ruota intorno: cucchiaini, coltelli, vassoi, piatti ecc. Nutella è stata citata nell'arte, con varie esposizioni, come quelle ad opera del gruppo Mistiche Nutelle esposte a Ferrara nel 1991 e incentrate sul celebre barattolo con ispirazione esplicitamente pop art; o Génération Nutella, a Parigi, nel 1996.

Ma l'apparizione più celebre e citata, in Italia, è forse quella del 1984 in Bianca in cui Nanni Moretti, depresso e nudo, in cucina, affonda coltello e dispiaceri in un mega barattolo di Nutella e poi spalma la crema sul pane. Il ruolo di conforto patemico della Nutella, che oggi fa parte del senso, e del sentire, comune, viene così affermato e celebrato. Ed è inscindibile dalla sua confezione: è

9 Sull'intertestualità cfr. Bernardelli (1997) e su intermedialità cfr. Ferraro (2001, 2003). Sul concetto di traduzione cfr. Jakobson (1963), Fabbri (2000), Dusi e Nergaard (2000). Sui casi di traduzione interdiscorsiva cfr. Marrone (2010), Fabbri e Pezzini (2012). 


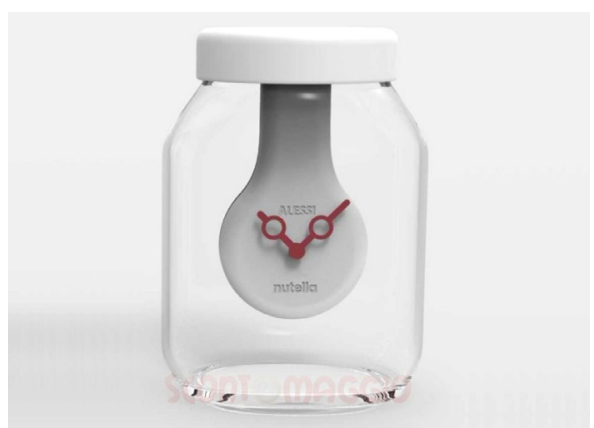

Figura 6. Nutella Clock di Alessi.

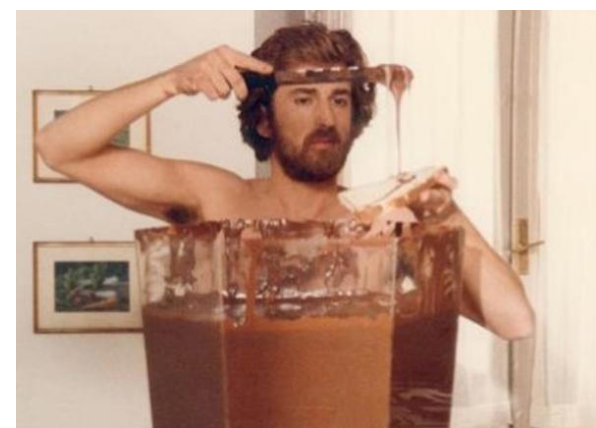

Figura 7. Nanni Moretti in Bianca, 1984.

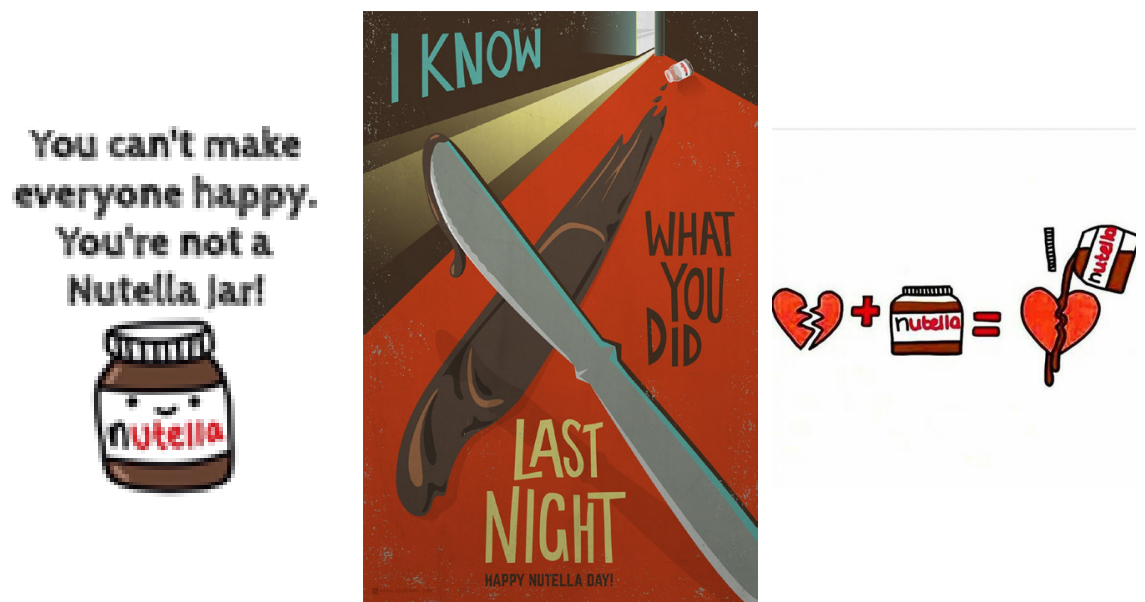

Figura 8. Alcuni dei meme che circolano su internet a proposito di Nutella e del suo ruolo patemico.

affondando cucchiai, dita, coltelli, biscotti o fette di pane dentro il barattolo che il ruolo patemico di Nutella raggiunge la sua massima efficacia (fig. 8).

Il caso della bottiglia dell'acqua Perrier è altrettanto emblematico. ${ }^{10} \mathrm{Nel}$ 1969 Perrier, già da tempo con un posizionamento di acqua di lusso ("lo champagne delle acque minerali” è lo storico pay-off) commissionò annunci ad alcuni artisti tra cui Dalì. Nel 1983 Andy Warhol ne aveva fatto delle litografie (fig. 9) sancendo così il marchio Perrier come segno rappresentativo della società, esattamente come Coca-Cola, Pepsi ed altri (Campbell, Chanel), anch'essi oggetti di consumo migrati e citati nel campo artistico. Perrier compare al cinema, ad esempio in Assassinio sull'Orient Express (1974, Sidney Lumet) dove il detective Hercule Poirot ne ordina una bottiglia, evidentemente a significare raffinatezza e francesità al tempo stesso. Ma i riferimenti più interessanti sono forse quelli ironici: in Il secondo tragico Fantozzi (1976, Luciano Salce), il ragioniere è costretto a bere dietro ordine del Mega Direttore, che ha accompa-

10 Come per la contour, sulla forma di questo oggetto circolano varie storie, una delle quali, è che la bottiglia derivi dalla sagoma degli strumenti ginnici che usava John Harmsworth, inventore del marchio, nei primi del Novecento. 


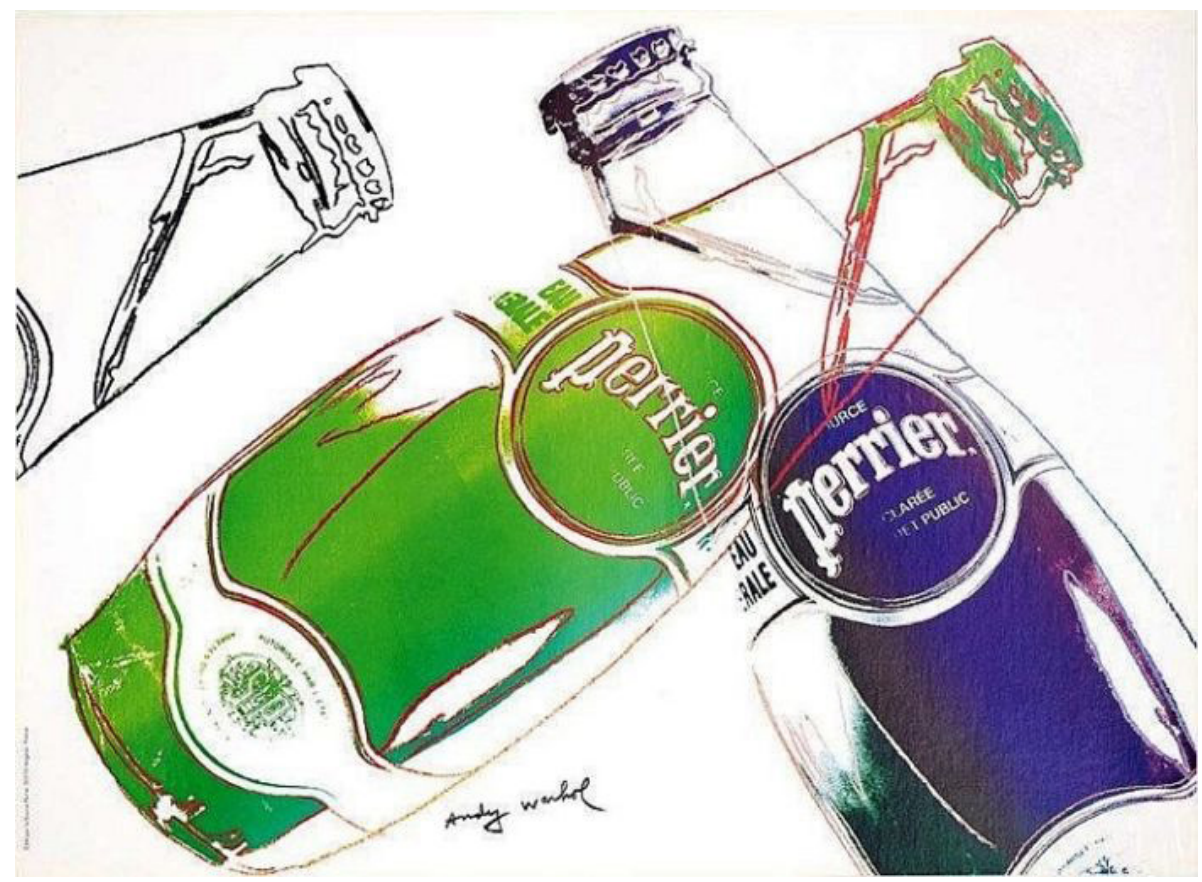

Figura 9. Andy Warhol, Perrier, 1983.

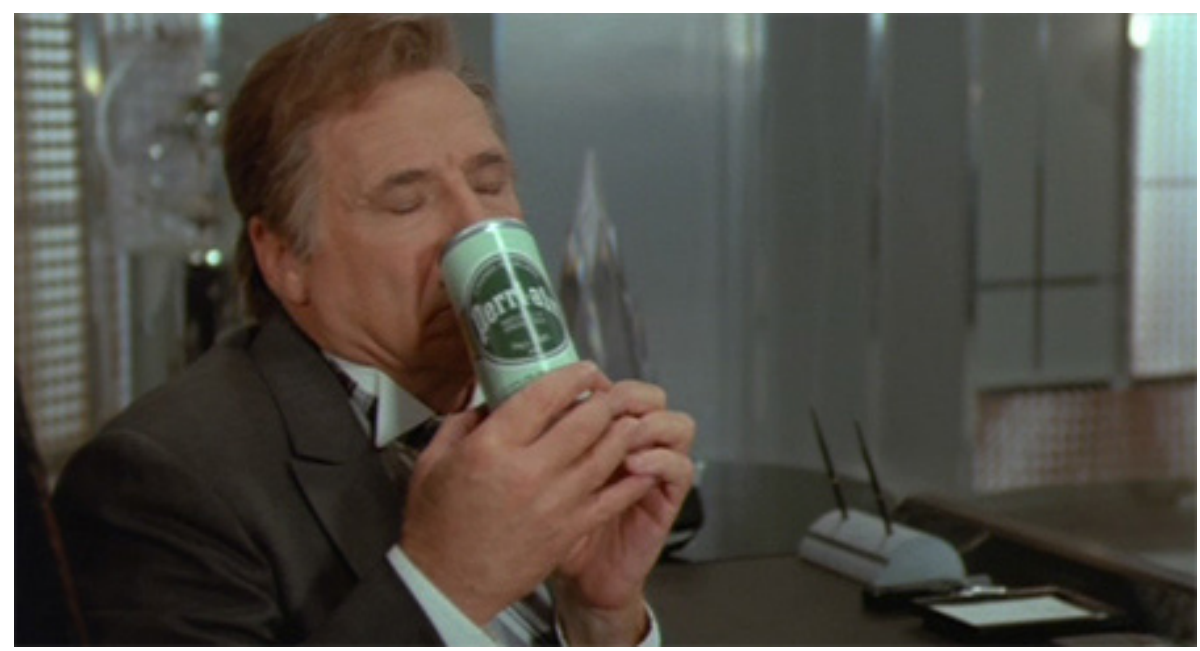

Figura 10. Balle spaziali, 1987.

gnato al casinò, quattro casse di acqua Bertier con effetti tragici e imbarazzanti; il nome è storpiato e tuttavia riconoscibile (Bertier richiama Perrier), ma individuiamo il riferimento a Perrier anche per altre caratteristiche, ovvero la frizzantezza e la quantità eccezionale di gas dell'acqua, la sagoma bombata della bottiglia, unica nel suo genere, e il colore verde del vetro. Anche in Balle Spaziali (1987, Mel Brooks), parodia delle varie pellicole di genere fantascienza di quegli anni, compare una lattina di acqua Perri-air da cui il presidente di Spaceballs, Scrocco, sniffa aria di nascosto (fig. 10). 
Vol 21, No 22 (April 2020) • DOI: 10.12977/ocula2020-20

Ilaria Ventura Bordenca | Il packaging come icona culturale? Processi di significazione e meccanismi intertestuali

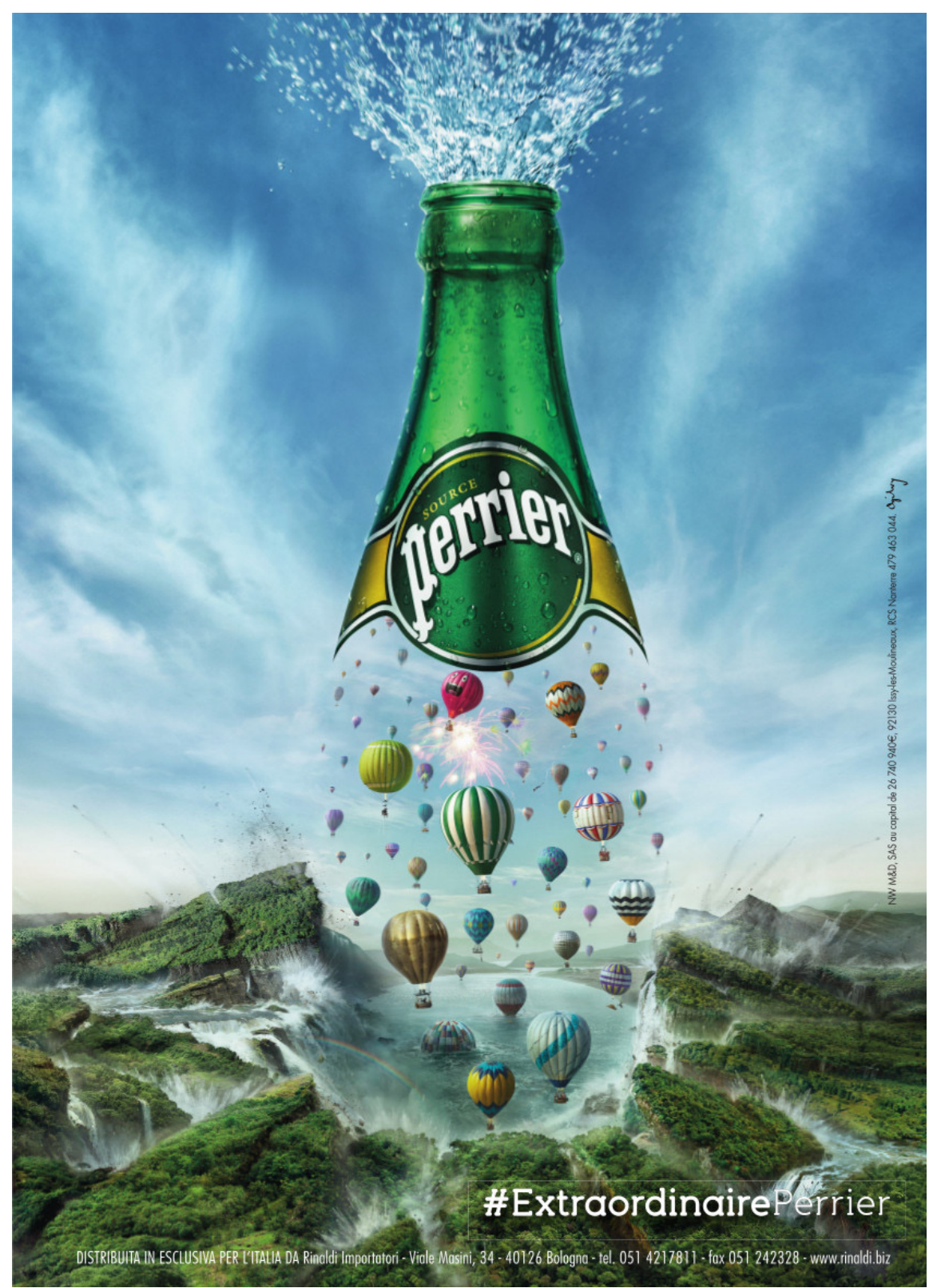

Figura 11. Annuncio Perrier, 2015.

Le citazioni sembrano ridicolizzare il marchio, puntando sull'eccesso di frizzantezza dell'acqua, ma in realtà finiscono per esaltarlo sia perché Perrier ha sempre puntato proprio sulla eccezionale quantità d'aria che contiene, sia perché lo ha fatto in maniere spesso ironiche ed equivoche. La figura della frizzantezza è inoltre un tratto caratteristico di quest'acqua, tradotto visivamente proprio dal packaging: la bottiglia è bombata come se contenesse una bolla d'aria, è tesa all'altezza della pancia (la parte centrale di una bottiglia), e la pubblicità non manca di evidenziarlo (fig. 11). 
Vol 21, No 22 (April 2020) • DOI: 10.12977/ocula2020-20

Ilaria Ventura Bordenca | Il packaging come icona culturale? Processi di significazione e meccanismi intertestuali

Questi giochi di citazioni, celebrazioni, esposizioni, rimandi più o meno parodici, riferimenti artistici più o meno seri, che influenza hanno nella costruzione dell'icona?

In primo luogo, occorre considerare che, una volta migrati dal discorso di brand in altri discorsi, gli oggetti in questione, ovvero i packaging, vengono trasformati, e al loro ritorno - perché al brand fanno inevitabilmente ritorno - non sono più gli stessi.

Vediamo in che modo. Prendiamo un caso apparentemente lontano, quello delle traduzioni televisive del personaggio letterario di Camilleri, il commissario Salvo Montalbano: nota Marrone (2018) che una volta finito nella fiction tv, dovendosi adattare alle necessità comunicative del mezzo, il personaggio del commissario, goffo e fisicamente pigro, diventa agile e capace di azioni rocambolesche, per trasformarsi poi anche nella successiva versione letteraria dove Camilleri lo rende più attivo di prima e somigliante al commissario televisivo. Insomma l'eroe mediatico, nel passaggio dalla carta allo schermo, quando aveva fatto ritorno alla narrazione cartacea, non era più lo stesso. La traduzione interdiscorsiva non lo aveva lasciato indenne, lo aveva trasformato.

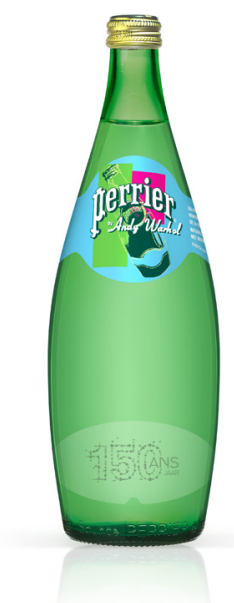

Figura 12. Bottiglia celebrativa per i 150 anni di Perrier: nell'etichetta l'immagine della bottiglia ritratta da Warhol.

Nel nostro caso, dopo che finiscono nelle maglie degli artisti, dei designer, dei registi, bottiglie, lattine e barattoli brandizzati, fanno ritorno nella pubblicità carichi e consapevoli: il brand riprende e rilancia il prodotto alla luce dei modi in cui è stato citato e usato da altri, si presenta come un Enunciatore astuto che riattualizza le riproposizioni altrui, a proprio uso e consumo. Avendo varcato i confini strettamente di marca, quel barattolo lì o quella bottiglia lì sono diventati altro, sono pieni di tutti quei significati che gli altri discorsi della cultura gli hanno fornito. ${ }^{11}$

11 In questo senso, non si tratta semplicemente della riproposizione dei linguaggi espressivi di altri campi che hanno influenzato la comunicazione pubblicitaria, come 
Vol 21, No 22 (April 2020) • DOI: 10.12977/ocula2020-20

Ilaria Ventura Bordenca | Il packaging come icona culturale? Processi di significazione e meccanismi intertestuali

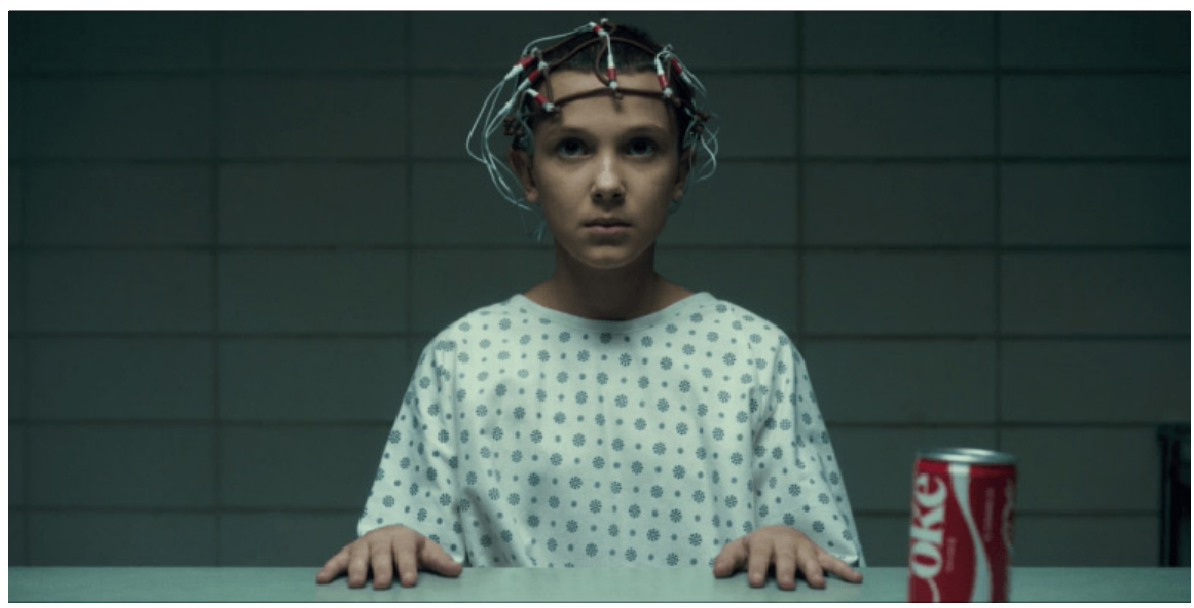

Figura 13. Lattina di Coca-Cola nella serie tv Stranger Things.

Ecco allora che nel 2013 Perrier disegna bottiglie ispirate alla pop art di Andy Warhol (fig. 12), compiendo non solo una citazione della pop art, ma soprattutto di sé stessa e del fatto di essere stata citata nell'arte. È l'icona che si riconosce in quanto tale. Negli anni Ottanta, Coca-Cola aveva fatto una mossa simile, chiamando Warhol a disegnare la copertina di Time per il lancio della New-Coke (poi ritirata): il marchio reingloba quelle istanze enuncianti inizialmente estranee, se non polemiche. Sulla stessa linea, di recente Coca-Cola è apparsa nella serie tv Stranger Things, ambientato proprio negli anni Ottanta, periodo in cui il product placement di Coca-Cola era diffusissimo (fig. 13). E Pepsi, anch'essa citata in tanti film anni Ottanta (al punto tale che esistevano due schieramenti di product placement concorrenti, o si era con Pepsi o con Coca-Cola) e nella pop art (come Pepsi-Cola Sign, di Oldenburg, 1961), ha riproposto in vari periodi le proprie citazioni: nei primi anni 90, con un annuncio per l'edizione limitata delle lattine con grafica ispirata proprio all'arte pop (fig. 14); nel 2013, con un annuncio con Beyoncé protagonista, ritratta a mo' delle serigrafie di Warhol (fig. 15); e poi nel 2015, anno in cui Marty McFly, del ciclo Ritorno al futuro, viene catapultato dal 1989 a bordo della DeLorean, Pepsi ha lanciato, in edizione speciale, le bottiglie futuristiche che compaiono nel Cafè 8o's di Ritorno al futuro 2 (fig. 16).

Quanto a Nutella, basti ricordare che, pur non essendo un oggetto che la pop art ha fatto in tempo a ritrarre (Nutella in quegli anni lì era appena nata), il barattolo si ritrova oggi riprodotto in tante immagini in serie con chiara, e

per esempio sostenuto da Grazioli a proposito dei rapporti tra pop art e pubblicità: «le immagini e i modi di Warhol sono entrati a tal punto del gusto diffuso, soprattutto giovalinile, da finire assunti nella comunicazione di massa come effetti di sicura riuscita: le sue scoperte passano insomma da una direzione all'altra della frontiera tra arte tout court e arte commerciale senza ulteriore deformazione o adattamento, così come sono, anfibie a tutti gli effetti, una sorta di compimento nell'iperrealtà dell'ideale dell'opera d'arte» (Grazioli 2001: 143). Dal nostro punto di vista, invece, entrambi i campi mutano di significato nel momento in cui ne viene oltrepassato reciprocamente il confine. 


\section{Our idea of pop art.}
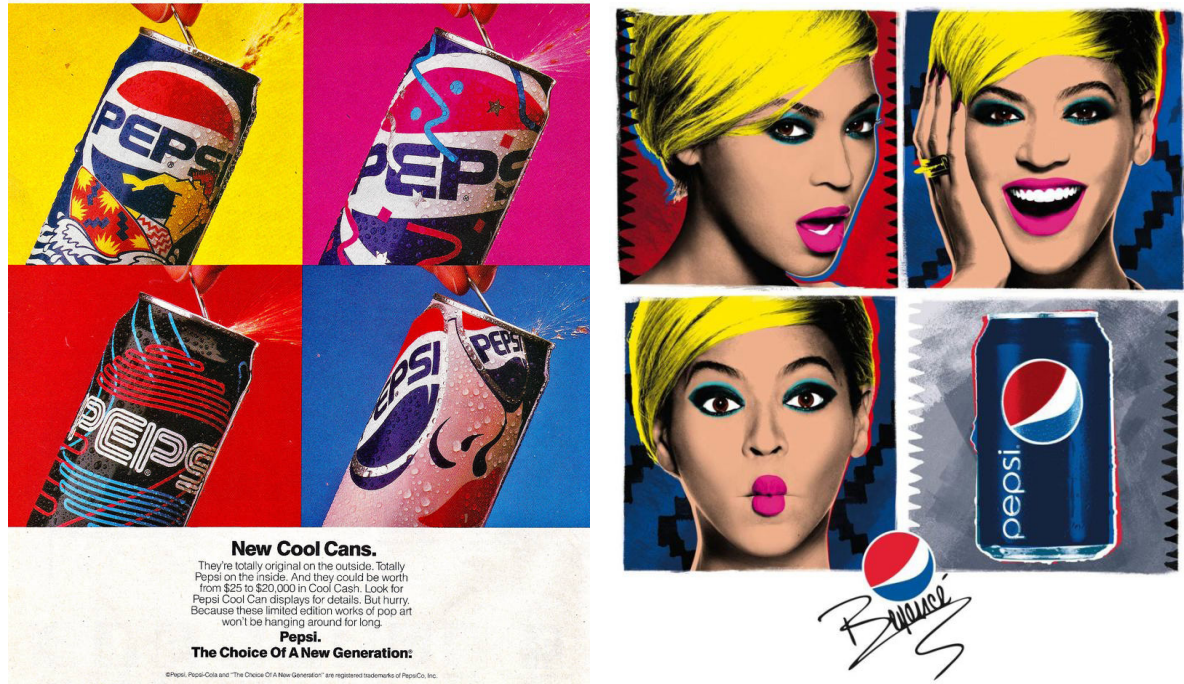

Figura 14. Annuncio per Pepsi limited edition, Figura 15. Annuncio Pepsi, 2013. anni Novanta.

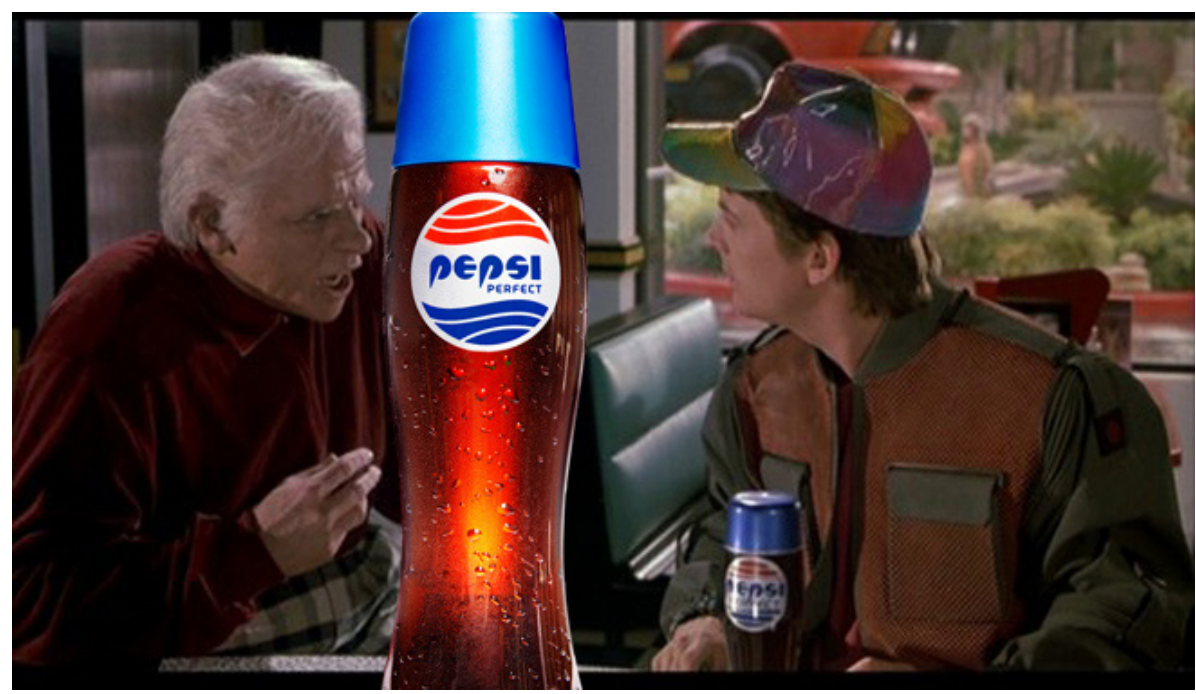

Figura 16. Pepsi limited edition (2015) in occasione dell'anniversario del product placement di Ritorno al futuro.

un po' stereotipa, ispirazione pop art. Questa imitazione delle forme espressive tipiche di un movimento artistico che ha utilizzato e rilanciato tanti prodotti di consumo che hanno conquistato il ruolo di icona culturale produce un ulteriore effetto citazionista e il riconoscimento di "icona".

Dusi e Spaziante scrivono, a proposito delle pratiche di replicabilità mediatica, che «un testo che si ricollega a un altro determina una scansione temporale tra un prima e un dopo, un processo di storicizzazione che è anche 
Vol 21, No 22 (April 2020) • DOI: 10.12977/ocula2020-20

Ilaria Ventura Bordenca | Il packaging come icona culturale? Processi di significazione e meccanismi intertestuali

un processo di produzione mitica perché tende a valorizzare il precedente» (Dusi e Spaziante 2006: 22). I processi di iconizzazione culturale che stiamo prendendo in esame sono l'esito di concatenazioni sintagmatiche di citazioni, che producono uno spessore discorsivo fatto di una molteplicità di piani comunicativi e semantici, e dalla moltiplicazione e sovrapposizione di Enunciatori: Coca-Cola in Stranger Things è sia una citazione dell'ambientazione anni Ottanta propria del tempo enunciato della serie, sia una citazione del product placement tipico del marchio in quegli anni, ed è quindi una sorta di meta-citazione, in modo tale che enunciato filmico ed enunciato del brand si sovrappongano in un inscatolamento piuttosto complesso. ${ }^{12}$

La comprensione delle reti intertestuali di riferimenti e la decifrazione dei passaggi precedenti tra un discorso e un altro presuppone nell'Enunciatario una competenza enciclopedica (Eco 1984): quando Pepsi pubblica l'annuncio "Our idea of pop art" si rivolge in primo luogo a quella fascia di pubblico che è in grado di riconoscere la citazione, esattamente come fa Coca-Cola o come fa Perrier con le sue edizioni limitate di ispirazione artistica. L'icona è insomma collettiva, condivisa, ma funziona solo in base a codici culturali che, piuttosto che unificare un pubblico, possono anche moltiplicarlo (cfr. Eco 1966).

\section{Icone culturali e usi collettivi}

L’iconizzazione degli oggetti di brand ci sembra essere un fenomeno di natura eminentemente enunciativa. Non sono pertinenti, per una analisi semiotica, la sua eziologia né la sua genealogia, cioè perché coinvolge certe marche piuttosto che altre, né qual è il punto d'inizio dei processi di riproposizione e intreccio tra i vari discorsi sociali nei quali i packaging che abbiamo citato si trovano coinvolti, e trasformati.

Importa il meccanismo di citazioni in sé, che, in quanto processo enunciativo con cui un testo migra in altri generi comunicativi e in altri contesti di produzione/ricezione, risemantizza il testo di partenza il quale torna prontamente e incessantemente a circolare per ulteriori nuove semantizzazioni. È la presa in carico, con l'autorialità, e l'autorevolezza, enunciative collegate, che fa variare il senso complessivo della produzione di un enunciato, la sua maggiore o minore ricchezza semantica, il taglio con cui lo si osserva.

La traduzione interdiscorsiva è il fenomeno che sta alla base della produ-

12 Marrone, a proposito del discorso di marca, parla della polifonia come di una caratteristica tipica della costruzione e della comunicazione del brand: «all'interno di un flusso discorsivo si produce una stratificazione, un accumulo più o meno gerarchicamente organizzato di piani enunciativi e di conseguenti contenuti enunciati, di voci e di messaggi che può avere esiti molto diversi e particolari» (2007: 231). Infatti, se la marca può essere riconosciuta come Autore consapevole, è anche vero che «essa è attraversata da enunciatori molteplici, da concatenamenti collettivi di enunciazione (Deleuze) che tendono a incrinarne l'autoralità e l'autorevolezza originaria, oppure se ben strutturati e gestiti possono apportare alla fonte discorsiva primaria il loro sapere, i loro contenuti, i loro valori di verità, il capitale di fiducia che, in altri campi, essi si sono progressivamente conquistati, rafforzando così l'istanza autoriale di partenza» (ivi: 232). 
Vol 21, No 22 (April 2020) • DOI: 10.12977/ocula2020-20

Ilaria Ventura Bordenca | Il packaging come icona culturale? Processi di significazione e meccanismi intertestuali

zione delle icone culturali. In particolare, seguendo Lévi-Strauss (1966) e l'idea che il mito sia l'insieme delle sue manifestazioni, i miti non sono «unità testuali ma macrotesti, o meglio, processi di migrazione da un testo all'altro, flussi traduttivi, catene interstuali senza inizio nè fine» (Marrone 2010: 57). Questo è proprio il caso dei packaging che assurgono a icone. Si ritrovano in una molteplicità di situazioni e generi, in una rete di relazioni, in un sistema di citazioni e rimandi di tipo sincronico (i vari casi di co-branding come quello di Nutella con Alessi) e diacronico (le confezioni ispirate alle citazioni d'autore del passato).

Secondo Lotman, ogni testo "crea" il proprio contesto e vive solo all'interno di un contesto socio-culturale, che a sua volta può diventare esso stesso un testo. Per esempio, per capire l'operazione di Pepsi con il packaging edizione speciale di Ritorno al futuro, occorre conoscerne il contesto, ovvero il riferimento al film e la presenza della lattina nel Bar del futuro: il testo stesso - il packaging e l'intera operazione di marketing - ha creato il suo contesto. A sua volta questo contesto (il film) è anche un testo vero e proprio. In questo modo, alla fine, l'oggetto-pack si sarà caricato di una serie composita e più o meno prevedibile di significati.

In generale, nel discorso di marca e nel processo di iconizzazione culturale di alcuni brand o di loro frammenti, si osserva, di fondo, una dialettica tra due tendenze opposte: una tendenza al controllo, al progetto, all'autorialità, da parte del marchio, che cerca di orientare strategicamente i rapporti con gli altri campi e gli altri attori sociali (si pensi alle sponsorship, alle pubbliche relazioni, ai co-branding, al product placement stesso, e a tutte quelle attività progettate per le relazioni con il mondo esterno, con gli altri marchi, con le istituzioni, con i personaggi della società ecc.); e una tendenza all'incontrollabilità dei contenuti, alla dispersione, all'imprevedibilità degli esiti semantici delle risposte della società (critiche del marchio, fail ripresi dai media, azioni di adbusting, usi imprevisti del marchio). Cioè, tutte quelle volte in cui il marchio viene nominato e utilizzato in modi non progettati dalla gestione centralizzata del brand si possono avere esiti più o meno felici.

Ma l'icona si caratterizza proprio perché è percepita come qualcosa di collettivo in quanto rappresentativa non tanto dei valori del brand ma di quelli di una certa comunità, di un certo gruppo, di un certo periodo: valori del brand e valori collettivi si fondono in un universo semantico inedito.

Nel campo del branding, l'iconizzazione può avvenire se la marca-autore perde un po' - o condivide un po' - del suo controllo a tutto vantaggio di un possesso semiotico collettivo.

La dispersione e la modificazione di un presunto senso originario di un testo che inizia a circolare in altri contesti non ne affievolisce il potere semiotico ma al contrario lo accresce: questa è un'altra caratteristica delle icone culturali (Fiorentino 2009). Ad esempio, quando Eco parla del processo di mitizzazione (1964) sostiene che anche nell'iconologia cristiana antica era impossibile distinguere quali archetipi visivi erano stabiliti dall'alto, istituzionalizzati dalla Chiesa, e quali invece emergevano dal basso, dal sentire popolare, di modo che le due direzioni mitopoietiche si confondevano in una produzione culturale unitaria. 
Vol 21, No 22 (April 2020) • DOI: 10.12977/ocula2020-20

Ilaria Ventura Bordenca | Il packaging come icona culturale? Processi di significazione e meccanismi intertestuali

Così che alla fine - ma non c'è una fine - $\mathrm{i}$ packaging presi in esame significano potenzialmente tutto ciò che li ha attraversati.

$\mathrm{Ci}$ può venire in aiuto la nozione di prassi enunciativa (Fontanille e Zilberberg 1998) che pone in evidenza la socialità intrinseca di qualsiasi comunicazione, ovvero il modo in cui usi, consuetudini, stereotipi, e altre abitudini comunicative collettive, si riverberano sul sistema linguistico sotto forma di virtualità possibili.

È proprio il caso delle icone culturali, per le quali, non solo non è detto che si possa individuare un Enunciatore unico e indiscutibile, ma l'enunciato che circola è proprio il risultato del contributo di molteplici istanze enunciative collettive che in qualche modo si fissano e si standardizzano, ritornando anche come abbiamo visto all'interno del discorso stesso della marca.

In questo modo, l'identità del brand e delle sue manifestazioni è il risultato di un bricolage culturale, nel senso che gli dà Floch (1995) a partire dalle considerazioni di Lévi-Strauss (1962): la produzione comunicativa è sempre il riuso di elementi della società e della cultura che sono già carichi di significati. Anche la pubblicità e il marketing si fondano su questo tipo di produzione di senso, sostiene Floch. Nel nostro caso, le migrazioni degli oggetti-confezioni in mondi altri, come quelli artistici, cinematografici, del design e così via, possono essere lette come operazioni di bricolage semiotico, e, a loro volta, i riutilizzi mediatici fatti dal brand di queste stesse migrazioni sono ulteriori forme di bricolage.

Per comprendere meglio il modo in cui questi processi di bricolage si articolano, stratificando, sovrapponendo o obliando diversi elementi della cultura, si può provare a far riferimento ai livelli di esistenza semiotica (virtuale, attualizzato, realizzato, potenziale). Secondo Fontanille e Zilberberg (1998) e il loro schema delle modalizzazioni esistenziali della prassi enunciativa, è possibile individuare i diversi processi di emersione e scomparsa di grandezze semiotiche allinterno di un sistema:

\begin{tabular}{|c|c|c|}
\hline Ascendenza & $\begin{array}{c}\text { Emergenza } \\
\text { virtuale }>\text { attuale }\end{array}$ & $\begin{array}{c}\text { Apparizione } \\
\text { attuale }>\text { realizzato }\end{array}$ \\
\hline $\begin{array}{l}\text { Declino } \\
\text { realizzato > potenziale }\end{array}$ & Distorsione & Fluttuazione \\
\hline $\begin{array}{c}\text { Scomparsa } \\
\text { potenziale }>\text { virtuale }\end{array}$ & Rimaneggiamento & Rivoluzione \\
\hline
\end{tabular}

Questo modello mostra la relazione e i passaggi tra i vari livelli di esistenza, visti da una prospettiva ascendente, cioè di progressiva realizzazione di un certo elemento, o al contrario, di progressiva decadenza, verso la virtualizzazione nel sistema. Non solo, ma le rispettive fasi di ascendenza (Emergenza e Apparizione) e decadenza (Declino e Scomparsa) vengono, in questo mo- 
Vol 21, No 22 (April 2020) • DOI: 10.12977/ocula2020-20

Ilaria Ventura Bordenca | Il packaging come icona culturale? Processi di significazione e meccanismi intertestuali

dello, ulteriormente messe in relazione, dando vita ad altre quattro possibili situazioni più complesse in cui i fenomeni di ascendenza e di decadenza sono compresenti.

Applicando questo modello ai meccanismi di senso delle icone culturali, che si basano, come abbiamo detto, su una dialettica tra dispersione e mantenimento, tra collettività e centralità autoriale, e su conseguenti continue trasformazioni semantiche, è possibile capire quali significati e quali temi emergono per un certo periodo, quali declinano, quali si mantengono costanti, quali ricompaiono. Non solo, come scrive Barthes «certi oggetti diventano preda della parola mitica per un momento, poi scompaiono, altri prendono il loro posto, accedono al mito. [...] si possono concepire miti molto antichi, ma non ne esistono di eterni» (Barthes 1957: 192, tr. it), ma è l'oggetto stesso che può variare significati in quanto «non c'è alcuna fissità nei concetti mitici: possono formarsi, alterarsi, sparire completamente» (ivi: 202, tr. it.). Aggiungiamo noi che all'interno dell'evoluzione di uno stesso oggetto mitico, più elementi, tratti culturali, grandezze semiotiche di vario tipo, possono alternarsi, mischiarsi, sovrapporsi, entrare in relazione in maniere differenti. ${ }^{13}$

Ad esempio, Pepsi con l'edizione speciale del packaging ispirato a Ritorno al futuro sta attualizzando un tema che era scomparso (il film Ritorno al futuro e tutti gli argomenti ad esso collegati come la fantascienza, la moda degli anni Ottanta, ecc.), passando dal declino (potenzializzazione) all'apparizione (realizzazione) attraverso le fasi di scomparsa (dalla potenzializzazione alla virtualizzazione, con cui il tema "anni Ottanta" si inabissa tra tutti i temi possibili a cui può attingere un brand) e di emergenza (dalla virtualizzazione all'attualizzazione, con cui inizia a intravedersi un trend culturale, come quello del ritorno del tema "anni Ottanta" a cui assistiamo oggi nella moda, in primis). La stessa cosa accade con le lattine di Coca-Cola in Stranger Things: emerge il collegamento con l'universo degli anni Ottanta come ricomparsa di un universo semantico già esistente e che era declinato, e che oggi ovviamente viene letto con uno sguardo diverso (primo fra tutti, quello nostalgico).

Gli annunci Pepsi dei primi anni Novanta e del 2013 apparentemente tematizzano lo stesso argomento, ovvero Pepsi e la pop art, ma lo fanno in maniere molto diverse già solo per il fatto che i due annunci compaiono a molti anni di distanza l'uno dall'altro, dopo vari cicli diversi di scomparsa e riemersione di tendenze. Nel primo, osserviamo una citazione delle opere pop art che hanno usato il marchio Pepsi, nel secondo non solo ritroviamo la citazione della citazione ma anche un riferimento agli anni Ottanta durante i quali furono progettate le lattine ispirate alla pop art.

Il modello della prassi enunciativa non solo permette di articolare più chiaramente come si organizza e sviluppa l'universo semantico di una certa icona culturale, ma aiuta anche a comprendere il processo di iconizzazione in diacronia e in sincronia.

13 Il modello della prassi enunciativa è stato applicato, tra gli altri, al campo del branding, per spiegare le varie forme di spessore enunciativo del discorso di marca (Marrone 2007) e nel campo del design, per comprendere i fenomeni dell'innovazione e della creatività nella progettazione degli oggetti (Mangano e Marrone 2010). 
Vol 21, No 22 (April 2020) • DOI: 10.12977/ocula2020-20

Ilaria Ventura Bordenca | Il packaging come icona culturale? Processi di significazione e meccanismi intertestuali

Infatti, ci permette di capire ad esempio il fatto che una stessa icona culturale può mutare parte dei propri significati nel corso del tempo: l'iconicità di Coca-Cola trent'anni fa non è la stessa di quella di oggi, al punto tale che oggi, in alcuni contesti, come abbiamo visto, la presenza dei packaging di Coca-Cola e Pepsi sta a significare proprio gli anni Ottanta, come isotopia tematica e figurativa.

Dall'altro lato, tale modello ci permette anche di vedere in che modo usi e abitudini di un certo oggetto si cristallizzano nella collettività e nell'immaginario di una certa icona, anche in contrasto con il progetto di marca che, in linea di principio, di quella stessa icona è il principale Enunciatore.

Ad esempio, l'isotopia di Nutella come "conforto patemico un po' bulimico" non è esplicitata nella comunicazione di marca - e non potrebbe starci oggi che siamo così attenti agli effetti salutari del cibo - ma fa parte dell'universo di senso collettivo che colleghiamo a questo prodotto. Il barattolo Pelikan di Nutella, cioè, si trova inserito in un fascio di significati in parte contrastanti: in certi discorsi, collettivi, diffusi, stereotipati, è il contenitore in cui tuffarsi in preda a nervosismi incontrollati (fig. 8), in quelli del brand è invece un oggetto esclusivamente euforico, anche dal forte valore estetico (si vedano i collegamenti tra Nutella e il design di cui abbiamo già fatto riferimento). In questo modo, temi e suggestioni passionali opposti sono in compresenza, a volte evidenti in alcuni contesti, a volte negati in altri (è lo stato della fluttuazione, secondo Fontanille e Zilberberg, che è dato dalla contemporaneità tra grandezze in declino e grandezze in apparizione).

\section{Apparenti contrasti: astrazione visiva ed effetto di iconicità}

Occorre esaminare un altro aspetto, quello propriamente visivo del packaging. Per il tipo di oggetti qui presi in considerazione l'identificazione e la riconoscibilità visive sono fondamentali. Innanzittutto perché sia per Coca-Cola che per Perrier, Nutella e Pepsi, il design del packaging è un elemento di differenziazione e di riconoscimento strategico.

Nella miriade delle trasformazioni e citazioni mediatiche, cosa resta di questi oggetti? Quanto vengono trasformati visivamente? È fondamentale che, nel corso delle varie citazioni e traduzioni interdiscorsive, si mantengano alcuni tratti visivi minimi, senza i quali l'identità si perderebbe del tutto.

In particolare la nostra ipotesi è che l'effetto di iconicità, nel senso di capacità di sintetizzare e convogliare un ricco e ampio universo di senso, può essere creato da una qualità inversa sul piano dell'espressione, cioè dall'uso di tratti minini visivi: ossia, dall'astrazione. Meno dettagli figurativi ci sono in un'immagine, più essa è in grado di comunicare un'identità forte, più il suo potere comunicativo si fa ricco. Ciò non vale sempre ma in particolare se tale immagine conserva certi tratti a seguito della pluralità delle sue trasformazioni.

Riprendiamo qui l'idea proposta da Floch (1993) riguardo la posizione di Greimas sulla densità figurativa. Per Greimas (1984), come si sa, più tratti specifici ci sono, più l'immagine è iconica. Floch sostiene che figuratività e 


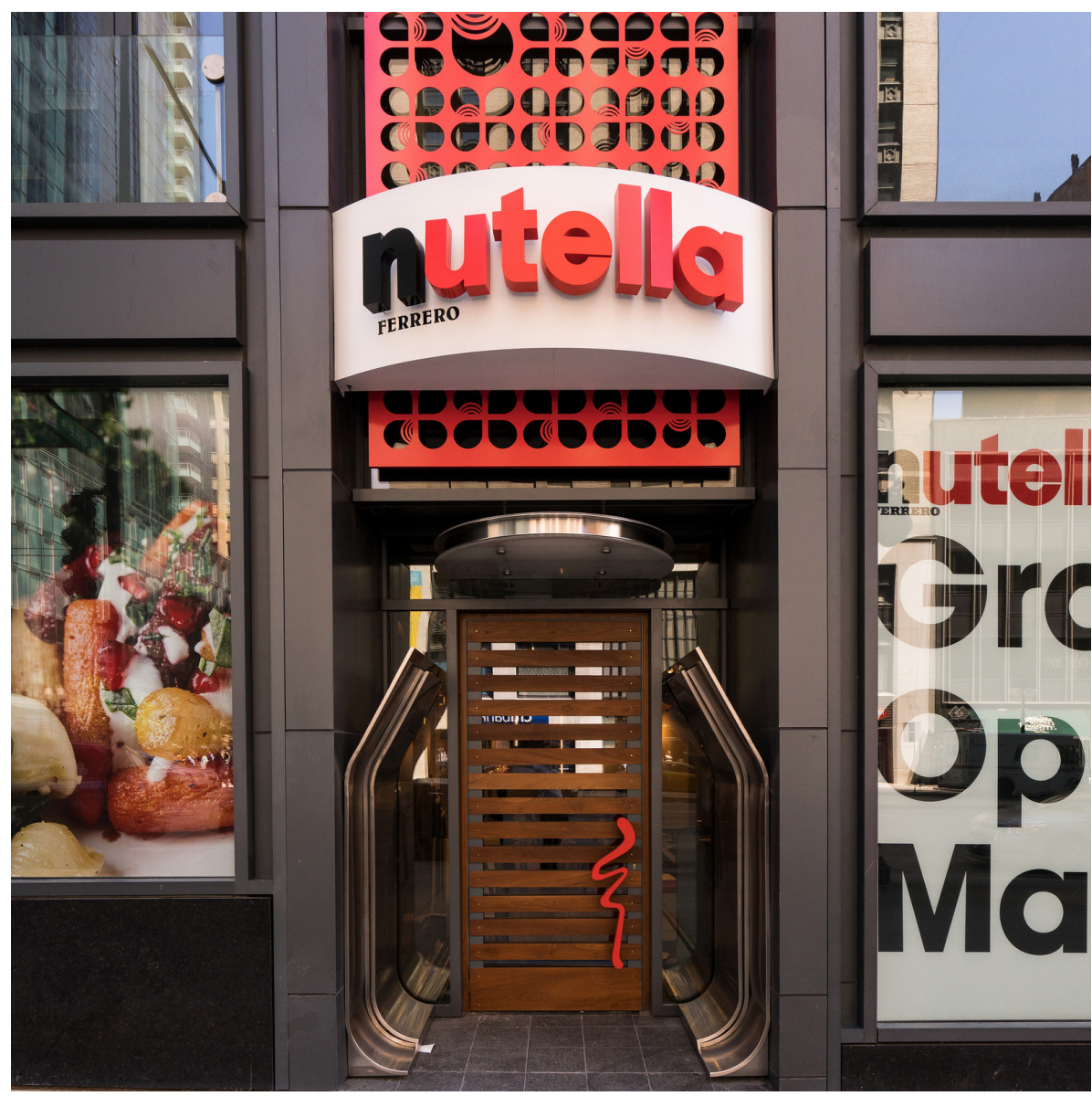

Figura 17. Il portone d’ingresso del Nutella Café di Chicago.

iconicità non siano, come per Greimas, differenze di grado, ma effetti completamente differenti per natura. Il figurativo riguarda la competenza dell'enunciatario e la sua capacità di riconoscere oggetti del mondo; l'iconico riguarda l'effetto di presenza, l'illusione referenziale. Secondo Floch, quindi l'icona non è per forza l'effetto di un eccesso di tratti visivi perché ci sono oggetti molto figurativi che non danno nessun effetto iconico, e viceversa immagini astratte che sono iconiche. ${ }^{14}$

Nel nostro caso, nell'insieme delle sue migrazioni, più l’immagine del packaging viene mutata, rimaneggiata, inserita in altri testi, più essa non perde identità, ma la acquisice, rafforzandola. Non solo nel senso che se ne arricchisce, assumendo una identità forte per bricolage, ma perchè a livello visivo

14 Floch parla di diversi tipi di astrazione: astrazione figurativa (la riduzione dei formanti figurativi a tratti visivi minimi necessari e sufficienti per il funzionamento dei formanti stessi); astrazione iconica (condizione minima per la creazione di un effetto di presenza) e astrazione plastica (che riguarda i discorsi visivi sulle condizioni minime della visione, ovvero sulle condizioni principali della messa in relazione tra mondo e soggetto attraverso prosodia e timismo). 


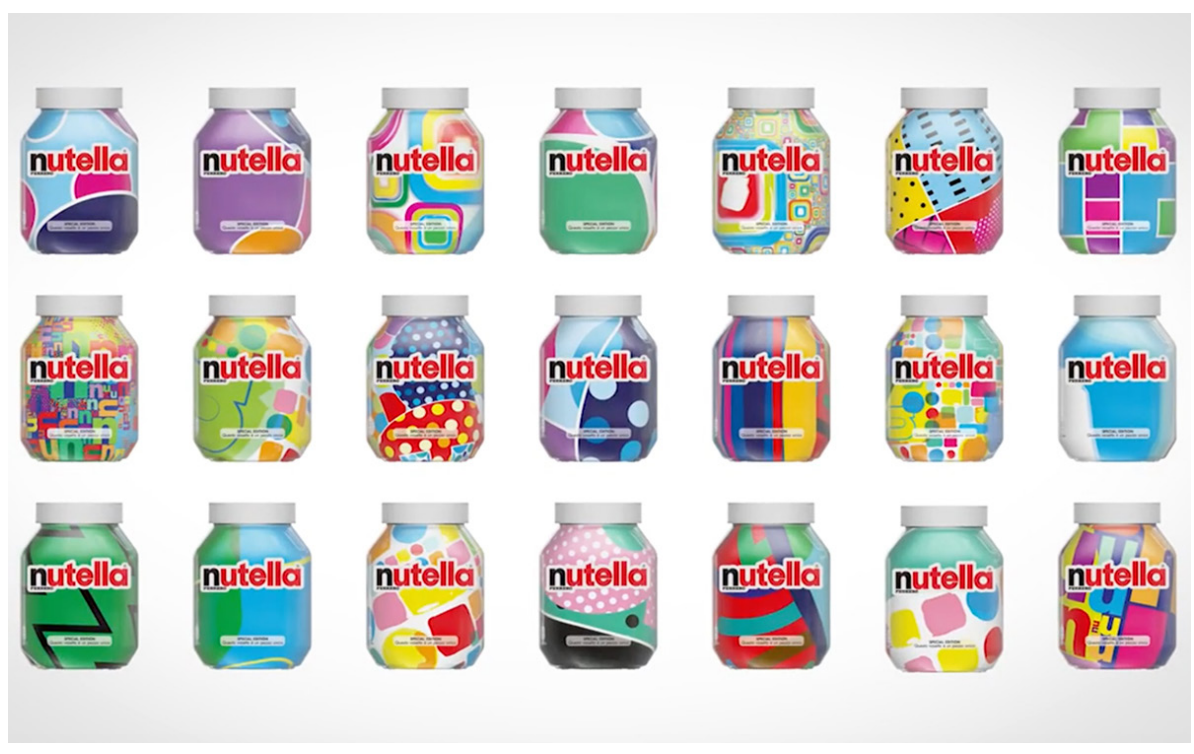

Figura 18. Nutella Seven Millions.

perde tratti superflui per mantere quelli che si costituiscono come invarianti. Perde densità figurativa, resta la sagoma, e paradossalmente, il packaging diventa così iconico.

Questa perdita di densità, e dunque il parallelo processo di astrazione visiva, procede per vari gradi. Ad esempio può basarsi su un grado minimo di riconoscimento, e coinvolgere solo la sagoma, il contorno. Ecco allora che il barattolo di Nutella si trasforma in un portone di ingresso per i Nutella Café (fig. 17), in un orologio (fig. 6), o in una cassettiera, ad esempio. Anche l'operazione "Nutella Seven Millions", con cui nel 2017 sono state messe in commercio confezioni dalla grafica multicolore, piuttosto che far perdere identità a Nutella, l'ha rafforzata perché proprio grazie alla differenze di colore si esaltano i tratti invarianti: la sagoma, il brand name e il tappo bianco, elementi che si mantengono costanti nella varie riproposizioni (fig. 18). Come accade nelle varie versioni speciali di Coca-Cola, quelle ad esempio firmate dagli stilisti di moda, e in quelle artistiche e cinematografiche: la sagoma della contour ha funzionato, insieme alla coppia cromatica rosso/bianco (non sempre presente però) e alla trasparenza del vetro. Per quanto riguarda Perrier, sembra che i tratti visivi fondamentali siano la sagoma bombata, il verde, il vetro. Warhol ad esempio li esalta tutti e tre.

Spesso poi i packaging diventano così importanti per l'identità dal brand che si trasformano in veri e propri logo: è il caso delle lattine Perrier al cui centro v'è la sagoma della bottiglia, come a dire che è la bottiglia in vetro il vero simbolo dell'acqua Perrier (fig. 19); e di Coca-Cola (fig. 20) che usa a mo' di logo non solo la contour della Coke classica, ma oggi anche le varie bottiglie della gamma (rosso classica, grigio light, nero zero, verde stevia).

Se l'icona è una immagine condensata che funziona per sintesi, il suo uso come logo ne rivela proprio tale capacità semiotica, in quanto il logo è proprio 


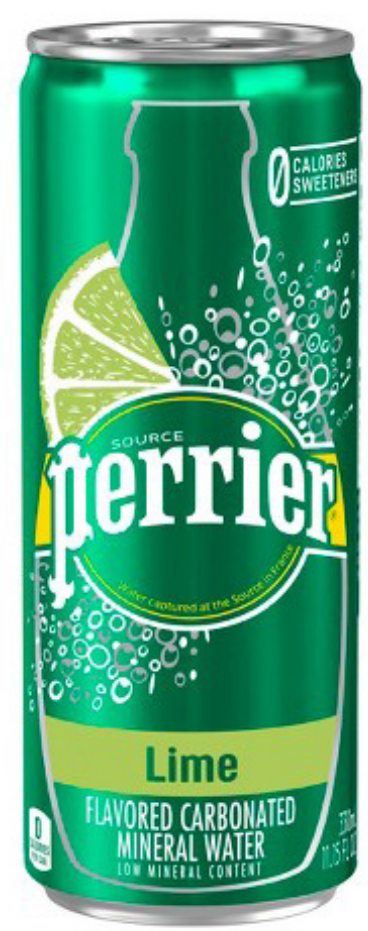

Figura 19. La bottiglia "dentro" la lattina.

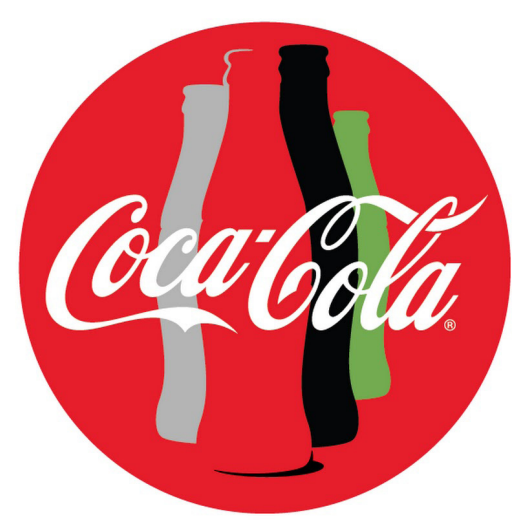

Figura 20. Le bottiglie diventano logo.

quel segno piccolo, a livello espressivo, che serve a comunicare, per condensazione, una serie complessa di significati, suggestioni, passioni e temi sul piano del contenuto.

\section{Conclusioni}

La questione dell'icona culturale, da un certo punto di vista, ricade all'interno delle problematiche dell'intertestualità e della traduzione, meccanismi di base per il funzionamento dei testi e, in generale, della cultura (Jakobson 1966; Lotman 1983, 1985; Fabbri 2000). Il caso dei packaging è soltanto esemplificativo della processualità che costituisce l'iconicità di un testo o di un insieme di testi. Le forme della creazione dell'icona, che sono in primo luogo di tipo enunciativo, cioè una presa in carico in discorsi altrui, sono comuni a tanti meccanismi comunicativi a livello sociale.

Quello che conta allora è l'assuzione ideologica dell'espressione "icona culturale": proprio perché il mito è storico, alcuni oggetti saranno icone per un periodo e lo sono solo perché vengono riconosciuti e nominati come tali. Infatti, nel caso dei packaging, siamo partiti dal senso comune, dal riconoscimento sociale di un ruolo culturale "altro" di questi oggetti, celebrati, nominati, citati in contesti enunciativi specifici come l'editoria, il giornalismo, l'arte, il cinema, ecc. E, partendo da questo livello di superficie, abbiamo provato a esplorare più in profondità quali sono i processi sottesi. 
Vol 21, No 22 (April 2020) • DOI: 10.12977/ocula2020-20

Ilaria Ventura Bordenca | Il packaging come icona culturale? Processi di significazione e meccanismi intertestuali

In generale, v'è una selezione di oggetti, in quanto elementi culturali, che più o meno spontaneamente vengono proposti in arte, cinema, spettacolo, design, perché dovrebbero sintetizzare una certa cultura; questa prassi va di pari passo con una indicalizzazione dell'icona tale per cui si dice "ecco, quella è l'icona di qualcosa"; questo riconoscimento, però, è già culturalizzato perché riflette un certo sistema di valori, ovvero una valenza, ed è già un punto di vista sulla icona stessa, ovvero un'icona si dà solo all'interno di una cultura che la assume come tale; e infine - ma non definitivamente - questo testo è trasformato e ritorna nel suo discorso originario, quello promozionale, caricato di nuovi sensi, pronti ad essere inglobati e rilanciati dai brand di appartenenza.

Tutto questo va di pari passo con un procedimento apparentemente paradossale: per essere comunicativamente forte e inglobare "pezzi" di una cultura, di un periodo, di una tendenza, il pack-icona deve perdere qualcosa, in un doppio senso. Deve perdere un po' di appartenenza al brand, per diventare un testo posseduto collettivamente, riempito dei significati più svariati, e deve anche perdere tratti visivi non pertinenti, per affermarsi come struttura visiva elementare, formata da elementi invarianti che ne garantiscono riconoscibilità. Per acquisire così quella capacità semiotica, propria dell'icona, di inglobare generosamente e divorare avidamente segni, restituendoli alla collettivià per ulteriori e potenzialmente infiniti usi.

\section{Bibliografia}

Barthes, Roland

1957 Mythologies, Paris, Seuil (tr. it. Miti d'oggi, Torino, Einaudi, 1974).

1982 L'Obvie et l'obtus, Paris, Seuil (tr. it. L'ovvio e l'ottuso, Torino, Einaudi, 1985).

Bernardelli, Andrea (a cura di)

1997 "The concept of Intertestuality", Versus, 77-78.

Bucchetti, Valeria

1999 La messa in scena del prodotto, Milano, FrancoAngeli.

2005 Packaging Design, Milano, FrancoAngeli.

Deni, Michela

2002 Oggetti in azione, Milano, FrancoAngeli.

Dusi, Nicola; Nergaard Siri (a cura di)

2000 "Sulla traduzione intersemiotica", Versus, 85-87.

Dusi, Nicola; Spaziante Lucio (a cura di)

2006 Remix remake: pratiche di replicabilità, Roma, Meltemi.

Eco, Umberto

1964 "Il mito di Superman", in Apocalittici e integrati, Milano, Bompiani.

1984 Semiotica e filosofia del linguaggio, Torino, Einaudi. 
Vol 21, No 22 (April 2020) • DOI: 10.12977/ocula2020-20

Ilaria Ventura Bordenca | Il packaging come icona culturale? Processi di significazione e meccanismi intertestuali

Fabbri, Paolo

1998 La svolta semiotica, Roma-Bari, Laterza.

2000 Elogio di Babele, Roma, Meltemi.

Fabbri, Paolo; Pezzini, Isabella (a cura di)

2012 Pinocchio. Nuove avventure tra segni e linguaggi, Milano, Mimesis.

Ferraresi, Mauro

1999 Il packaging. Oggetto e comunicazione, Milano, FrancoAngeli.

Ferraro, Guido

2001 "Entrare nel www", in Lombardi A. (a cura di), Il dolce tuono, Milano, FrancoAngeli.

2003 “Sonny per Adidas: un'epopea intermediale”, in Semprini A. (a cura di), Lo sguardo sociosemiotico, Milano, FrancoAngeli.

Fiorentino, Francesco (a cura di)

2009 Icone culturali d'Europa, Macerata, Quodlibet.

Floch, Jean-Marie

1993 "L'opposition abstrait/figuratif mérite-t-elle qu'on prenne de graves options méthodologiques en sémiotique visuelle?", Versus, 65/66, "Del Visibile" a cura di O. Calabrese.

1995 Identités visuelles, Paris, PUF (tr. it. Identità visive, Milano, FrancoAngeli 1997).

Fontanille, Jacques; Zilberberg, Claude

1998 Tension et signification, Liège, Mardaga.

Grazioli, Elio

2001 Arte e pubblicità, Milano, Mondadori.

Greimas, Algirdas Julien

1984 “Sémiotique figurative et sémiotique plastique”, Actes Sémiotiques.

Documents, 60, Paris (tr. it. "Semiotica figurativa e semiotica plastica", in

Fabbri P., Mangano D., a cura di, La competenza semiotica, Roma, Carocci, 2012).

Heyer, Paul

2012 Titanic Century: Media, Myth and the Making of a Cultural Icon, London, Praeger.

Jakobson, Roman

1963 Saggi di linguistica generale, Milano, Feltrinelli.

Landowski, Eric; Marrone, Gianfranco

2002 La società degli oggetti, Roma, Meltemi.

Lévi Strauss, Claude

1962 Il pensiero selvaggio, Milano, Il Saggiatore.

1966 Antropologia strutturale, Milano, Il Saggiatore.

Lotman, Juri M.; Ivanov, I.V.; Uspenskij, B.A.

1973 "Tesi per una semiotica delle culture", in Lotman J.M., Tesi per una semiotica delle culture, a cura di F. Sedda, Roma, Meltemi. 
Vol 21, No 22 (April 2020) • DOI: 10.12977/ocula2020-20

Ilaria Ventura Bordenca | Il packaging come icona culturale? Processi di significazione e meccanismi intertestuali

Lotman, Juri M.

1980 Testo e contesto, a cura di S. Salvestroni, Roma-Bari, Laterza.

1983 La cultura e l'esplosione, Milano, Feltrinelli.

1985 La semiosfera, a cura di S. Salvestroni, Venezia, Marsilio.

Mangano, Dario

2009 Semiotica e design, Roma, Carocci.

2014 Ikea, Doppiozero, Miti d’oggi.

2019 Ikea e altre semiosfere, Milano, Mimesis.

Mangano, Dario; Marrone, Gianfranco

2010 "Princìpi di semio-design. Forme dell'innovazione e teoria del progetto", Ticonzero, 106.

Marrone, Gianfranco

2007 Il discorso di marca, Roma-Bari, Laterza.

2010 L'invenzione del testo, Roma-Bari, Laterza.

2018 Storia di Montalbano, Palermo, Edizioni Antonio Pasqualino.

Mattozzi, Alvise

2006 Il senso degli oggetti tecnici, Roma, Meltemi.

Panofsky, Erwin

1955 Meaning in the Visual Arts. Papers in and on Art History, New York, Doubleday (tr. it. Il significato nelle arti visive, Torino, Einaudi, 1962)

Pilditich, James

1973 The Silent Salesman, Londra, Business Book.

Ventura, Ilaria

2014 Che cos'è il packaging, Roma, Carocci.

Ilaria Ventura Bordenca è titolare di un assegno di ricerca (M-FIL/O5) presso il Dipartimento Culture e Società dell'Università degli studi di Palermo. È dottore di ricerca in Disegno Industriale, Arti figurative e Applicate. È autrice di Che cos'è il packaging? (Carocci 2014). Ha insegnato Percezione e Comunicazione Visiva, Packaging Design e Merchandising, Marketing presso i corsi di laurea in Scienze della Comunicazione dell'Università degli Studi di Palermo. Ha pubblicato diversi saggi sui temi del cibo, della dietetica, del brand, del packaging e tiene regolarmente lezioni e seminari su food packaging, semiotica del gusto, semiotica del design. 